\title{
NORMAL DIVISION ALGEBRAS OVER ALGEBRAIC NUMBER FIELDS NOT OF FINITE DEGREE*
}

\author{
BY A. A. ALBERT
}

1. Introduction. If $R$ is the field of all rational numbers and if $\xi_{1}, \ldots, \xi_{n}$ are ordinary algebraic numbers, then the field $\Omega=R\left(\xi_{1}, \cdots, \xi_{n}\right)$ of all rational functions with rational coefficients of $\xi_{1}, \cdots, \xi_{n}$ is an algebraic number field of finite degree (the maximum number of linearly independent quantities of $\Omega$ ) over $R$. It has recently been proved $\dagger$ that every normal simple algebra over such a field $\Omega$ is cyclic. In particular it has been shown that every normal division algebra of order $n^{2}$ (degree $n$ ) over $\Omega$ is cyclic and has exponent $n$.

In the present note I shall give an extension of the above results to normal division algebras over any algebraic number field $\Lambda$. I shall prove that all normal division algebras over $\Lambda$ are cyclic and with degree equal to exponent but shall give a trivial example showing that the theorem corresponding to the above on normal simple algebras is false. The problem of the equivalence of normal division algebras over $\Lambda$ will also be discussed.

2. Cyclic Algebras. Let $F$ be any non-modular field and let $Z$ by cyclic of degree $n$ over $F$. Then $Z$ possesses a generating automorphism

$S:$

$$
z \longleftrightarrow z^{S},
$$$$
\left(z \text { in } Z, z^{S} \text { in } Z\right) \text {, }
$$

such that every automorphism of $Z$ is one of $S^{0}=S^{n}=I, S$, $S^{2}, \cdots, S^{n-1}$. The algebra $A$ of all quantities

$$
\sum_{i=0}^{n-1} z_{i} y^{i}, \quad(z \operatorname{in} Z),
$$

is a cyclic algebra with multiplication table

$$
y^{n}=\gamma \text { in } F, y^{e} z=z^{S^{e}} y^{e}, \quad(e=0,1, \cdots),
$$

* Presented to the Society, October 28, 1933.

$\dagger$ See the paper by H. Hasse and myself in the Transactions of this Society, vol. 34 (1932), pp. 722-726, for the normal division algebra theorem. The theorem for normal simple algebras follows from Hasse's Theorem 6 of his Transactions paper, vol. 34 (1932), pp. 171-214. 
for every $z$ of $Z$. Evidently $A$ is uniquely defined by $Z, S, \gamma$, and thus we write

$$
A=(Z, S, \gamma)
$$

Let $F$ be contained in any larger field $K$. Then

$$
A_{K}=(Z, S, \gamma)_{K}
$$

is the algebra with the same basis and constants of multiplication as $A$, but over $K$.

If $A_{K}$ is a division algebra, then so evidently is $A$. But then $Z_{K}$, which is the algebra with the same basis and constants of multiplication as the field $Z$, but over $K$, is a field and in fact is evidently cyclic of degree $n$ over $K$. Evidently $A=\left(Z_{K}, S, \gamma\right)$ over $K$.

TheOREM 1. Let $A=(Z, S, \gamma)$ over $F, F<K$, and let $A_{K}$ be a division algebra. Then $A_{K}$ is the cyclic algebra $\left(Z_{K}, S, \gamma\right)$ over $K$.

3. The Determination of Algebras over $\Lambda$. Let $\Lambda$ be any nonmodular field whose quantities are all algebraic numbers and let $A$ be a normal division algebra of order $m=n^{2}$ over $\Lambda$, If $u_{1}, \cdots, u_{m}$ are a basis of $A$, then $u_{i} u_{j}=\sum^{\prime} \gamma_{i j k} u_{k}$ with $\gamma_{i j k}$ in $\Lambda$. But then $\gamma_{i j k}$ are all algebraic numbers, so that $L=R\left(\gamma_{111}, \cdots\right.$, $\left.\gamma_{i j k}, \cdots, \gamma_{m m m}\right)$ is algebraic of finite degree.

The linear set $B=\left(u_{1}, \cdots, u_{m}\right)$ over $L$ is evidently an algebra of order $m$ over $L$. If in particular $u_{1}=1$, the modulus of $A$, then $u_{1}$ is the modulus of $B$. Evidently $A=B_{\Lambda}$.

If $B$ contains any divisors of zero, then these quantities are in the division algebra $A$, a contradiction. Hence $B$ is a division algebra.

Let $B$ contain a quantity $k=\sum \lambda_{i} u_{i}, \lambda_{i}$ in $L$, which is commutative with every quantity of $B$. In particular $k u_{i}=u_{i} k$, so that $k\left(\sum \mu_{i} u_{i}\right)=\left(\sum \mu_{i} u_{i}\right) k$ for $\mu_{i}$ any quantities of the field $\Lambda$. But $A$ is normal, so that $k$ is a multiple of the modulus $u_{1}$ of $A$ by a quantity of $\Lambda$. Hence $k=\mu u_{1}=\sum \lambda_{i} u_{i}$. Since the $u_{i}$ are linearly independent in $\Lambda$, we have $\mu=\lambda_{1}, k$ is a multiple of $u_{1}$ by a quantity of $L$, and $B$ is normal.

The normal division algebra $B$ of degree $n$ over $L$ is thus* a cyclic algebra $(Z, S, \gamma)$ over $L$. The basis, $\left(u_{i}\right)$, of $A$ is linearly

* By the result already quoted on normal division algebras over $\Omega$. 
expressible with coefficients in $L$ in terms of the basis of $B=(Z, S, \gamma)$ in its cyclic form, so that in fact $A=(Z, S, \gamma)_{\Lambda}$. By Theorem 1 we have the following result.

TheOREM 2. Let $A$ be a normal division algebra of degree $n$ over an algebraic number field $\Lambda$ not of finite degree. Then there exists a sub-field $L$ (of $\Lambda$ ) of finite degree and a cyclic algebra, $B=(Z, S, \gamma)$, over $L$ such that $A=\left(Z_{\Lambda}, S, \gamma\right)$ over $\Lambda$, where $Z_{\Lambda}$ is a cyclic field of degree $n$ over $\Lambda$. Hence $A$ is cyclic.

4. The Exponent of Algebras A. Suppose that the algebra $A$ of Theorem 2 has exponent $\rho<n$. Then $A^{\rho}$ is well known to be equal to $M^{\rho-1} \times\left(Z_{\Lambda}, S, \gamma^{\rho}\right)$, where $M$ is a total matric algebra. But $A^{\rho}$ is a total matric algebra; hence $\left(Z_{\Lambda}, S, \gamma^{\rho}\right)$ is also. Hence $\gamma^{\rho}$ is the norm $N(c)$ of a quantity $c$ of $Z_{\Lambda}$.

Let $Z=L(x), Z_{\Lambda}=\Lambda(x)$, so that $c=\sum c_{i} x^{i}$, where the $c_{i}$ are in $\Lambda$. The field $L=L\left(c_{0}, \cdots, c_{n-1}\right)$ is algebraic of finite degree. Moreover, if $B=(Z, S, \gamma)$, then evidently $Z_{0}=L_{0}(x), B_{0}=\left(Z_{0}, S, \gamma\right)$ over $L_{0}$, is contained in $A$ and hence is a cyclic division algebra. But $B_{0}^{\rho}=\left(Z_{0}, S, \gamma^{\rho}\right) \times M^{\rho-1}$ is a total matric algebra, since $\gamma^{\rho}=N(c)$, where $c$ is in $Z_{0}$.

The exponent of $B_{0}$ of degree $n$ over $L_{0}$ is known to be $n$ since $B$ is a cyclic division algebra over $L_{0}$, which is algebraic of finite degree. Hence $\rho \geqq n$, a contradiction.

THEOREM 3. The exponent of any normal division algebra over $\Lambda$ is its degree.

5. On the Equivalence of Algebras over $\Lambda$. Let $A=\left(Z_{\Lambda}, S, \gamma\right)$ and $C=\left(Y_{\Lambda}, T, \delta\right)$ over $\Lambda$ be normal division algebras. Then $Z$ and $\gamma$ are obtained with respect to a field $L_{1}$ defined by $A, Y$, and $\delta$ with respect to $L_{2}$ defined by $C$. If $L$ is the composite of $L_{1}$ and $L_{2}$, then we may evidently take $L$ as the common field of Theorem 2 for both algebras $A$ and $C$. Hence $A=(Z, S, \gamma)_{\Lambda},(Z, S, \gamma)$ a normal division algebra over $L, C=(Y, T, \delta)_{\Lambda},(Y, T, \delta)$ also a normal division algebra over $L$.

The algebra $A$ is equivalent to the algebra $C$ if and only if $A \times C^{-1}=(Z, S, \gamma) \times\left(Y, T, \delta^{-1}\right)$ is a total matric algebra. But, as is well known, $(Z, S, \gamma) \times\left(Y, T, \delta^{-1}\right)=(X, R, \epsilon) \times M$, where $M$ is a total matric algebra and $(X, R, \epsilon)$ is a uniquely determined cyclic algebra. Evidently $A \times C^{-1}$ is total matric if and only if $(X, R, \boldsymbol{\epsilon})_{\Lambda}$ is total matric. For $A \times C^{-1}=M \times(X, R, \boldsymbol{\epsilon})_{\Lambda}$. 
But then $\epsilon=N(c)$, where $c$ is in $X_{\Lambda}$. As before there exists a sub-field $L_{0}$ of $\Lambda$ of finite degree such that $c$ is in $X_{L_{0}},(X, R, \epsilon)_{L_{0}}$ is total matric. But then $(Z, S, \gamma)_{L_{0}}$ is equivalent to $(Y, T, \delta)_{L_{0}}$. The converse is obvious and we have proved this theorem.

Theorem 4. Let $A$ and $C$ be normal division algebras of degree $n$ over $\Lambda$, an algebraic field not of finite degree, so that $A=\left(Z_{\Lambda}, S, \gamma\right)$, $C=\left(Y_{\Lambda}, T, \delta\right)$, where $B=(Z, S, \gamma), D=(Y, T, \delta)$ are cyclic over the same sub-field $L$ of finite degree of $\Lambda$. Then $A$ and $C$ are equivalent if and only if there exists a sub-field $L_{0}>L$ of $\Lambda$ such that $L_{0}$ has finite degree and the algebras $B_{L_{0}}$ and $D_{L_{0}}$ are equivalent.

The above theorem essentially reduces the problem of the equivalence of normal division algebras over $A$ to the corresponding problem (already solved*) for algebras over fields of finite degree, and to a consideration of the sub-fields of $\Lambda$ of finite degree.

6. Normal Simple Algebras over $\Lambda$. In this section we shall show trivially that there exist non-cyclic normal simple algebras over an algebraic field $\Lambda$. We take $\Lambda$ to be the field of all constructible (with ruler and compass) numbers, extended by $i=(-1)^{1 / 2}$. That is, we take $\Lambda$ to consist of all numbers obtained from rational numbers by a finite number of rational operations and extractions of square roots.

Evidently any equation $x^{2}=c, c$ in $\Lambda$, is reducible in $\Lambda$ since $c^{1 / 2}$ is also in $\Lambda$. But then there exist no cyclic algebras of degree two over $\Lambda$. Hence the total matric algebra of degree two over $\Lambda$, a normal simple algebra, is non-cyclic.

The Institute for Advanced Study

* See Hasse, loc. cit. 